Voix et Images

volxetimages

\title{
Papineau, " celui qui n’écrit pas »
}

L'inscription de l'homme politique dans l'histoire littéraire québécoise

Papineau, “The One Who Doesn't Write”

Writing the Politician into Quebec's Literary History

Papineau, 'el que no escribe'

La inscripción del hombre político en la historia literaria quebequense

\section{Jonathan Livernois}

Volume 42, numéro 1 (124), automne 2016

URI : https://id.erudit.org/iderudit/1038591ar

DOI : https://doi.org/10.7202/1038591ar

Aller au sommaire du numéro

\section{Éditeur(s)}

Université du Québec à Montréal

\section{ISSN}

0318-9201 (imprimé)

1705-933X (numérique)

Découvrir la revue

Citer cet article

Livernois, J. (2016). Papineau, « celui qui n'écrit pas » : l'inscription de l’homme politique dans l'histoire littéraire québécoise. Voix et Images, 42(1), 97-111. https://doi.org/10.7202/1038591ar

\section{Résumé de l'article}

Cette étude s'attache à la présence de Louis-Joseph Papineau dans les manuels et essais d'histoire littéraire du Québec ainsi qu'aux incidences idéologiques de sa représentation en tant qu'écrivain. Elle s'attache, notamment, au Manuel d'histoire de la littérature canadienne-française (1918 et 1939) et à l'Histoire de la littérature canadienne (1930) de $\mathrm{M}^{\mathrm{gr}}$ Camille Roy; à l'Histoire de la littérature canadienne-française (1957) de Samuel Baillargeon; au Manuel de littérature canadienne-française (1967) de Roger Duhamel ; à l'Histoire de la littérature française du Québec, en quatre volumes (Beauchemin, 1967-1969), dirigée par Pierre de Grandpré ; à La littérature québécoise depuis ses origines (1974-1997) de Laurent Mailhot ; à La vie littéraire au Québec (premier volume paru en 1991) ; et à l'Histoire de la littérature québécoise (2007) de Michel Biron, François Dumont et Élisabeth Nardout-Lafarge. Nous verrons s'y jouer les stratégies alternatives d'intégration et de refus de la charge symbolique et politique de «l'écrivain " Papineau. Cela permettra de mieux comprendre pourquoi ces stratégies sont étonnamment récurrentes au fil de l'histoire littéraire, malgré les tours et détours idéologiques et historiographiques du $\mathrm{xx}^{\mathrm{e}}$ siècle. 


\title{
PAPINEAU, "CELUI QUI N'ÉCRIT PAS "
}

L'inscription de l'homme politique dans $l^{\prime}$ histoire littéraire québécoise ${ }^{1}$

$$
++
$$

\author{
JONATHAN LIVERNOIS \\ Université Laval/Centre de recherche interuniversitaire \\ sur la littérature et la culture québécoises (CRILCQ)
}

Depuis les travaux de Micheline Cambron et de Marilyn Randall ${ }^{2}$, on ne saurait méconnaître les rapports complexes de la fiction et de l'histoire lorsque cellesci abordent l'épisode des rébellions de 1837 et 1838. Dans cette perspective, j'ai récemment dégagé, en portant une attention particulière à la fictionnalisation de Louis-Joseph Papineau dans les œuvres de Louis Fréchette ${ }^{3}$, trois stratégies qui permettaient au poète d'intégrer l'homme politique tout en repoussant, paradoxalement, sa charge symbolique et politique. On aura vu à l'œuvre une désolidarisation ou un isolement du personnage, magnifié mais sorti du cercle de sa communauté; une dépolitisation suivie d'une "repolitisation » fictionnelle qui désamorce le radicalisme de sa pensée; un dessèchement rapide du souvenir, qui permet de «momifier» le personnage, de l'écraser sous le poids de l'histoire, lui conférant un statut de spectre plus ou moins menaçant. À la lecture des mises en récit de l'histoire littéraire du Québec, depuis le Manuel d'histoire de la littérature canadiennefrançaise (1918) de Camille Roy à l'Histoire de la littérature québécoise (2007) de Michel Biron, François Dumont et Élisabeth Nardout-Lafarge, force est de constater que ces stratégies ne se limitent pas à l'usage exclusif de Fréchette. Elles y occupent aussi une place importante lorsque vient le temps de présenter Papineau comme écrivain ou acteur de la vie littéraire, pour reprendre le vocabulaire de La vie littéraire au Québec.

La volonté de repousser la charge radicale et revendicatrice de Papineau s'explique aisément pour le début du siècle, tandis que le clergé, en délicatesse avec l'homme politique héritier des Lumières, avait la mainmise sur l'enseignement, les

1 Cette étude participe d'un projet de recherche subventionné par le Fonds de recherche du Québec - Sociêté et culture, dont je reconnais l'apport. Je remercie également Marie-Andrée Beaudet et Yvan Lamonde pour leurs lectures judicieuses, ainsi qu'Emmanuelle Germain et Julia Hains, assistantes de recherche, pour leur collaboration.

2 Par exemple: Micheline Cambron, «Du "Canadien errant" au "Salut aux exilés" : l'entrecroisement de l'histoire et de la fiction", Études françaises, vol. XXVII, n 1, printemps 1991, p. 75-86; Marilyn Randall, «Plus patriote que ça... Fictions du Patriote 1837-1981", Voix et Images, vol. XXVI, n 3, printemps 2001, p. 536.

3 Voir Jonathan Livernois, «Le Papineau de Fréchette: l'exproprié de l'histoire», Études littéraires, vol. XLV, no 2, été 2014, p. 203-204. 
manuels et les essais d'histoire littéraire ${ }^{4}$. Il est plus difficile de comprendre la persistance des stratégies d'isolement et de momification du passé après les années 1950 et 1960, tandis que le recul du religieux, les changements historiographiques et les nouvelles avenues méthodologiques en études québécoises auraient pu changer la donne. Les traits idéologiques d'hier semblent pourtant s'être incrustés jusque dans nos lectures contemporaines.

C'est pour mieux comprendre les raisons de cette persistance que je m'attacherai ici à huit manuels ou histoires littéraires, dont la plupart ont été longuement étudiés par Karine Cellard dans Leçons de littérature ${ }^{5}$ : le Manuel d'histoire de la littérature canadienne-française (1918 et 1939) et l'Histoire de la littérature canadienne (1930) de $\mathrm{M}^{\mathrm{gr}}$ Camille Roy; Littérature canadienne-française (1957) de Samuel Baillargeon; le Manuel de littérature canadienne-française (1967) de Roger Duhamel; l'Histoire de la littérature française du Québec, en quatre volumes (Beauchemin, 19671969), dirigée par Pierre de Grandpré; La littérature québécoise depuis ses origines (1974, largement remanié et augmenté en 1997) de Laurent Mailhot; La vie littéraire au Québec (depuis 1991); et l'Histoire de la littérature québécoise (2007) de Michel Biron, François Dumont et Élisabeth Nardout-Lafarge. Le portrait est nécessairement partiel: les manuels scolaires et les essais d'histoire littéraire sont ici associés, sans distinctions préalables, tandis que d'autres ouvrages - des manuels scolaires actuels, des anthologies, etc. - auraient pu se retrouver dans cette liste. Mais je tiens le pari que ces huit titres, bornes reconnues de l'histoire littéraire au Québec, sauront tisser un fil suffisamment solide pour qu'on puisse mieux comprendre la rémanence des thèmes et des stratégies d'inclusion/exclusion depuis bientôt cent cinquante ans.

\section{PAPINEAU, LE MAUVAIS ÉCRIVAIN ?}

La question se pose d'emblée: peut-on véritablement considérer Louis-Joseph Papineau comme un écrivain? Certes, il est l'auteur d'adresses et de discours reproduits dans les journaux, mais aussi de l'Histoire de l'insurrection du Canada, d'abord parue en mai 1839 dans La revue du progrès politique, social et littéraire de Louis Blanc, reprise le mois suivant dans La revue canadienne de Ludger Duvernay, à Burlington, au Vermont. Aussi, pendant son exil en France, au cours des premières années de la décennie 1840, Papineau prépare — sans jamais l'écrire - une histoire

4 «[D]urant les années 1920, 69 \% des nouveaux manuels sont édités par des communautés religieuses. Même si la part du marché qu'elles détiennent décroît régulièrement par la suite, ces dernières, prises dans leur ensemble, demeurent les principaux producteurs d'ouvrages pédagogiques jusque dans les années 1950, alors qu'elles impriment, éditent et signent encore la moitié de tous les manuels francophones mis en circulation au Québec.» Paul Aubin, "L'édition du manuel scolaire au Canada français», Carole Gerson et Jacques Michon (dir.), Histoire du livre et de l'imprimé au Canada, t. III: De 1918 à 1980, Montréal, Presses de l'Université de Montréal, 2007, p. 252.

5 Dans Leçons de littérature. Un siècle de manuels scolaires au Québec (Montréal, Presses de l'Université de Montréal, coll. «Nouvelles études québécoises, 2011, 387 p.), Karine Cellard ne s'est pas directement intéressée aux questions politiques, mais a quand même réfléchi à l'élaboration d'une identité narrative (au sens de Ricœur) et aux valeurs participant de l'écriture des manuels scolaires consacrés à l'histoire littéraire du Québec. 
du Canada en copiant et faisant copier six mille pages tirées des archives du ministère de la Marine ${ }^{6}$. On verra là un rappel du geste de certains Anciens, comme Thucydide et Polybe, successivement "hommes d'action, engagés dans la politique, historiens ensuite quand commence pour eux le temps de l'exil $^{7}{ }^{\prime}$.

Cela ne suffit pas, bien sûr, à faire de Papineau un écrivain. Mais, à une époque où il n'y a pas encore de processus d'autonomisation du champ littéraire, et où les discours littéraires ne sont pas toujours bien cloisonnés, fruits du travail d'écrivains qui sont, le plus souvent, des polygraphes ${ }^{8}$, Papineau n'est pas exclu d'emblée. D'autant que, comme le rappellent Denis Saint-Jacques et Alain Viala, au Québec, «l'autonomie du littéraire se fait d'abord avec l'autonomie politique, et non contre le politique ${ }^{9}$ ». Papineau pourrait être du lot. Et il le sera. Mais en tant que mauvais écrivain. Laurent-Olivier David écrira ainsi, un an après sa mort: "À l'exemple de beaucoup d'orateurs, M. Papineau est un médiocre écrivain; son style diffus, rempli d'incidences interminables, qui se croisent et se débattent avec fracas, rend la lecture de ses écrits peu agréable ${ }^{10}$.» Le propos est, bien sûr, relayé au $x^{e}$ siècle: Lionel Groulx, par exemple, considérera que «[r]ien n'est moins lisible ni ne se prête moins à l'analyse qu'un article de journal ou un discours du Papineau d'après $1848^{11}$ ».

On peut croire que c'est Hector Fabre, en 1866, lors d'une conférence consacrée à la littérature canadienne et présentée à la Literary and Historical Society of Quebec, qui ouvre le bal:

Une immense auréole d'orateur entoure le nom de M. Papineau, mais en relisant ses discours, sans doute mal rapportés, et où d'éternelles redites ne contribuent pas peu à faire paraître interminables des phrases déjà longues par elles-mêmes, on ne conçoit de son éloquence qu'une idée bien au-dessous de 1'admiration qu'elle inspirait à ses contemporains ${ }^{12}$.

Dès lors, si - et il faut insister sur le conditionnel — on concède à Papineau le titre d'écrivain, ce sera aussitôt pour en faire un mauvais écrivain, incapable de faire passer à l'écrit ce que sa parole aurait eu - peut-être - d'exceptionnel. Les deux stratégies évoquées plus haut, celle de la momification rapide et de l'isolement, fonctionneront

6 Voir notamment Yvan Lamonde, Signé Papineau. La correspondance d'un exilé, Montréal, Presses de l'Université de Montréal, 2009, p. 236-244.

7 François Hartog, Evvidences de l'histoire. Ce que voient les historiens, Paris, Gallimard, coll. «Folio. Histoire», 2007 [2005], p. 26.

8 Voir, à ce propos, Yvan Lamonde, «L'écrivant polygraphe: terra incognita de la littérature québécoise du $19^{e}$ siècle», Julien Goyette et Claude La Charité (dir.), avec la collaboration de Catherine Broué, JosephCharles Taché polygraphe, Québec, Presses de l'Université Laval, coll. «Cultures québécoises», 2013, p. 19-37.

9 Denis Saint-Jacques et Alain Viala, «Ā propos du champ littéraire: histoire, géographie, histoire littéraire», Bernard Lahire (dir.), Le travail sociologique de Pierre Bourdieu. Dettes et critiques, Paris, La Découverte, coll. «La Découverte poche. Sciences humaines et sociales», 2001, p. 69-70.

10 Laurent-Olivier David, L'Honorable Ls.-Jos. Papineau, Montréal, Typographie Geo. E. Desbarats, 1872, p. 25.

11 Lionel Groulx, Notre maître, le passé, deuxième sêrie, Montréal, Librairie Granger frères limitée, 1936, p. 191.

12 Hector Fabre, "On Canadian Literature», Transactions of the Literary and Historical Society of Quebec. Session of 1865-6, Québec, Middleton \& Dawson/The Gazette, 1866, p. 99. 
de pair: la parole de Papineau ne passera pas l'épreuve du temps et ne deviendra qu'un souvenir de moins en moins certain. Quand Fabre évoque «l'admiration qu'elle inspirait à ses contemporains", il ne faut pas oublier que Papineau n'est pas encore mort, et que le conférencier est lui-même un contemporain de l'homme, même si, né en 1834, il est beaucoup plus jeune que lui. Pourtant, tout se passe comme si Papineau était repoussé très loin dans le passé, avec les Anciens auxquels un Louis Fréchette, par exemple, ne manquera pas de le rattacher ${ }^{13}$. Si on reconnaît tout de même la grandeur de la parole de l'orateur (" [l']immense auréole d'orateur entoure le nom de M. Papineau "), c'est pour mieux l'isoler, le plus souvent, dans ces manuels et essais d'histoire littéraire, à l'extérieur de la littérature. Cette exclusion s'explique bien: Papineau, homme de paroles, se distingue des plus jeunes patriotes, comme Thomas Chevalier de Lorimier, par exemple, dont les écrits sont pétris de thèmes romantiques et littéraires ${ }^{14}$. Papineau appartient à un autre temps, au «régime normatif des Belles-Lettres ${ }^{15}$ ", où prévalent "les vieux canons des belles lettres ${ }^{16}$ » (il est vrai que plusieurs de ses discours sont construits à la manière des modèles antiques appris au collège classique ${ }^{17}$ ) ainsi que les prises de parole des «orateurs du peuple ${ }^{18}$ ». C'est ainsi que Berthelot Brunet peut écrire, en 1946, dans son iconoclaste Histoire de la littérature canadienne-française:

Il [Papineau] vivait à l'époque du romantisme, mais l'on peut dire qu'il se montra plutôt pré-romantique. Les livres arrivaient tellement en retard chez nous!

N'en est-il pas toujours ainsi, ou guère s'en faut? Les orateurs ne saisissent que l'éloquence écrite d'une époque révolue. L'orateur le plus avancé retarde toujours, comme une grosse horloge ${ }^{19}$.

La frontière du littéraire permettra d'endiguer, d'une certaine manière, la parole de Papineau. Avec une étonnante régularité, les manuels et les essais d'histoire littéraire québécois, par une sorte de mouvement alternatif, incluront et excluront du même

13 Voir Jonathan Livernois, «Le Papineau de Fréchette: l'exproprié de l'histoire», p. 191-192.

14 «On peut également se demander en quoi et comment la génération des patriotes est romantique. En réalité, non seulement l'engagement politique et les idéaux qu'ils proclament sont associés à la pensée nationale et libérale romantique (autorité du peuple, droit des peuples à disposer d'eux-mêmes, liberté d'expression, etc.), mais les textes laissés par plusieurs d'entre eux regorgent [...] de thèmes, d'images et de procédés relevant de cette esthétique.» Marie-Frédérique Desbiens, La plume pour l'épée. Le premier romantisme canadien (1830-1860), thèse de doctorat, Québec, Université Laval, 2005, f. 136-137.

15 Jacques Rancière, La parole muette. Essai sur les contradictions de la littérature, Paris, Fayard, 2010, p. 13.

16 Jacques Rancière, Les noms de l'histoire. Essai de poétique du savoir, Paris, Éditions du Seuil, coll. «La librairie du Xxe siècle», 1992, p. 106.

17 À ce propos, Marc André Bernier écrit: «À la différence de ce que prétendent la plupart de ses biographes, c'est dès l'année 1801, à Québec et non pas à Montréal, que Papineau fut formé à la rhétorique sous la direction d'un disciple de Plessis, Pierre-Jacques Bossu (1770-1802). Inscrite dans le prolongement de certaines des leçons essentielles de l'enseignement qu'il reçoit, sa prose si volontiers néocicéronienne, voire "néoclassique" et déjà "académique", s'organise précisément en larges périodes imitant le mouvement et l'ampleur de la prose de l'orateur romain.» Marc André Bernier, "Patriotes et orateurs: de la classe de rhétorique à l'invention d'une parole rebelle», Voix et Images, vol. XXVI, n³, printemps 2001, p. 498-515.

18 Jacques Rancière, Les noms de l'histoire, p. 106.

19 Berthelot Brunet, Histoire de la littérature canadienne-française, Montréal, Éditions de l'Arbre, 1946, p. 20-21. 
coup le personnage: présent par la parole, laquelle ne devient qu'une sorte de souvenir évanescent, Papineau est toujours placé sur la face externe du littéraire. Cette exclusion a des implications idéologiques, on s'en doute bien: en d'autres temps et en d'autres lieux, Barthes et Foucault ont bien montré comment la consolidation d'une mémoire littéraire permettait d'endiguer des paroles et d'étouffer des voix ${ }^{20}$.

C'est dans cette perspective que Lucie Robert écrivait ceci, en 1982, à propos du manuel de $\mathrm{M}^{\mathrm{gr}}$ Camille Roy:

On ne peut pas dire que Voltaire ou Louis-Joseph Papineau soient tout à fait inoffensifs et conformes aux valeurs des manuels dans lesquels ils occupent une place si importante. [...] Il faut bien reconnaître que les normes mêmes qu'édictent ou infléchissent les classes dominantes ont pour résultat de sélectionner des écrits qui souvent leur nuisent. Et, si le discours de la littérature a souvent pour effet de vider un écrit de sa force politique au profit d'une reconnaissance esthétique de la valeur de son écriture (Voltaire, les orateurs de la Révolution française, Papineau), il a aussi pour effet de mettre à la disposition des étudiants auxquels il s'adresse à travers les manuels des textes qui servent de base à une prise de conscience politique (Vallès, Camus, Aragon). [...] La négociation [entre l'Église et la bourgeoisie] impose le compromis d'admettre dans le corpus des écrits qu'on peut récupérer en marge, et déplacer les valeurs (la langue de Voltaire, l'art oratoire de Papineau, l'absurde de Camus) devient une nécessité pour éviter le pire ${ }^{21}$.

Dans le cas de Papineau, il est vrai que le contenu politique est occulté, mais la contrepartie - la reconnaissance esthétique de ses écrits - n'est pas pour autant présente. S'il est intégré dans ces histoires littéraires, c'est sans doute pour «éviter le pire» et canaliser, autant que faire se peut, ce qu'il peut représenter comme risque d'une "prise de conscience politique».

\section{PAPINEAU, ÉCRIVAIN : AVANT 1960}

On peut partir, justement, du Manuel de $\mathrm{M}^{\text {gr }}$ Camille Roy. Dans l'édition de 1918, la figure de Papineau se retrouve dans deux sections («L'éloquence politique» et «Journalisme») du premier chapitre («La prose») de la "Deuxième période. Premiers développements 1820-1860». Dans la première section, où l'on retient la figure du tribun Papineau, le portrait n'est pas particulièrement négatif: on dit de l'homme politique qu'il «a incarné pendant longtemps les aspirations les plus fières de ses concitoyens du Bas-Canada». Aucun mot, par contre, sur un ouvrage comme l’Histoire

20 Roland Barthes, «Réflexions sur un manuel» [1971], Le bruissement de la langue. Essais critiques IV, Paris, Éditions du Seuil, coll. «Points», 1993 [1984], p. 51. Voir aussi, bien sûr, Michel Foucault, L'ordre du discours, Paris, Gallimard, 1971, 81 p.

21 Lucie Robert, Discours critique et discours historique dans le Manuel d’histoire de la littérature canadienne de langue française de $M^{g r}$ Camille Roy, Québec, Institut québécois de recherche sur la culture, coll. «Edmondde-Nevers», 1982, p. 33. 
de l'insurrection du Canada ${ }^{22}$, comme si le politicien n'était qu'homme de paroles. Papineau y est également associé à Auguste-Norbert Morin et à Louis-Hippolyte La Fontaine, ses collaborateurs d'avant 1837 devenus ses adversaires politiques après son retour d'exil en 1845 - Roy ne souffle mot de ces ruptures et donne plutôt à voir une succession ininterrompue de luttes et de victoires ${ }^{23}-$, qui ont «moins de puissance oratoire», mais "une parole où l'argumentation ferme s'accompagne d'un style net et sobre ${ }^{24} »$. La sobriété a-t-elle une plus grande valeur que la puissance? On ne peut répondre à cette question qu'en abordant la section qui suit, consacrée à Étienne Parent. La représentation de cette figure a de nombreux avantages pour celui qui, comme $\mathrm{M}^{\mathrm{gr}}$ Camille Roy, cherche à raconter l'histoire tout en insistant sur une attitude de modération qui serait représentative du tempérament canadien-français (et qui, a posteriori, justifierait le régime confédératif): victime des rébellions (il est emprisonné de décembre 1838 à avril 1839), Parent a pourtant rejeté la stratégie patriote, refusant de suivre Papineau dans une voie jugée radicale. Camille Roy le convoque pour en faire le père du journalisme au Canada français. À la puissance des discours, Roy oppose la modération de l'écrit:

\begin{abstract}
À l'époque même où haranguaient Papineau, Morin et La Fontaine, un journaliste écrivait dans sa gazette où s'exprimait aussi, avec une éloquence tour à tour impérieuse, ironique, âpre ou légère, l'âme même du peuple canadien-français. L'on peut dire qu'Étienne Parent a tout aussi bien, et plus longtemps, et plus fidèlement que Papineau, représenté la pensée profonde de ses compatriotes.
\end{abstract}

Plus loin, dans la même section:

Au surplus, Étienne Parent montra toujours dans l'exposé et la défense de ses principes politiques une calme et suffisante modération. Il n'aima jamais les excès, les excès de parole pas plus que les excès de l'action. Et bien qu'il ait longtemps bataillé à côté de Papineau, [...] il ne put suivre le chef des patriotes. Il s'en sépara quand il lui sembla que Papineau allait sortir des voies de la prudence et de la légalité ${ }^{25}$.

Quels sont les traits qui distinguent les deux hommes, dont les destins sont séparés avec netteté autour de 1837? Chose certaine, les qualités de Parent sont mises en relief par les défauts de Papineau, et vice versa. Dans les deux passages, on associe la figure de Parent à la polyvalence de l'éloquence, à la pérennité du propos juste, à la modération. Surtout, et c'est là que tout semble se jouer, l'un parle et l'autre écrit. Ce que Parent fixe dans sa «gazette» est durable, mesuré, limité en quelque sorte par la

22 Louis-Joseph Papineau, «Histoire de l'insurrection du Canada en réfutation du rapport de lord Durham», La Revue canadienne, première livraison, juin 1839, 35 p.

23 Cette façon d'envisager la période des rébellions et ses suites est assez commune dans l'historiographie clérico-nationaliste. Voir Yvan Lamonde et Jonathan Livernois, Papineau. Erreur sur la personne, Montréal, Boréal, 2012, p. 32-37.

24 Camille Roy, Manuel d'histoire de la littérature canadienne-française, Québec, Imprimerie de l'Action sociale limitée, 1918, p. 25.

25 Ibid., p. 25 et p. 28-29. 
page, tandis que Papineau ne connaît que la démesure, que la logorrhée qu'engendre une parole indomptée et illimitée.

Dans l'édition de 1939 du Manuel, Papineau est toujours la principale figure de «l'éloquence politique», mais il ne côtoie plus Parent. Roy y évoque plus clairement ce qu'il définit comme sa violence verbale, tout en pointant du doigt l'isolement (stratégie de la désolidarisation ou de l'isolement du personnage) dans lequel cette violence l'a cantonné: "Après 1830, son patriotisme brûlant, irrité, l'emporta vers des excès où ne voulurent pas le suivre quelques-uns de ses meilleurs partisans. Des intransigeances maladroites, et aussi des violences de paroles devaient l'entraîner sur une pente qui aboutit à l'insurrection de $1837^{26}$.» La parole a, en elle-même, de lourdes conséquences.

Dans l'esprit du manuel de $\mathrm{M}^{\mathrm{gr}}$ Roy, la parole de Papineau n'est pas, non plus, particulièrement originale. Dans l'Histoire de la littérature canadienne de 1930, comme dans l'édition de 1939 du Manuel, d'ailleurs, Roy parle d'une parole convenue, apprise à l'école, devine-t-on: «Volontiers elle s'inspire des lieux communs de la rhétorique et des souvenirs classiques». Il ajoute: «Il y a dans ses discours des inégalités de composition, des développements où s'abandonne sans assez de retenue une verve abondante, et où fléchit la discipline de la pensée ${ }^{27}$. " La parole a ainsi, derrière cette abondance, quelque chose de figé. On reconnaît là, comme le rappelait Lucie Robert, la "critique à fondements "classiques" qui ne s'y limite pas» de l'ecclésiastique, laquelle confère à la rhétorique "un sens péjoratif: de grandes envolées vides de $\operatorname{sens}^{28} »$.

Double stratégie, donc: Papineau est isolé par une parole mortifère, qui n'a rien d'original (en témoigne l'insistance de $\mathrm{M}^{\mathrm{gr}}$ Camille Roy sur les lieux communs d'une rhétorique qu'il n'apprécie guère ${ }^{29}$ ); sa parole est projetée dans un passé qui momifie rapidement (stratégie du dessèchement du souvenir), comme si la parole était glacée, sans suite, et fausse - parce que prise dans les rets d'une rhétorique qui tourne à vide.

Littérature canadienne-française de Samuel Baillargeon, ouvrage préfacé par le chanoine Groulx, paru pour la première fois en 1957 puis réédité jusqu'en 1972, prend, pour ainsi dire, le relais du manuel de Camille Roy. Comme le rappelle Karine Cellard, le rédemptoriste y maintient «l'ordre social et des certitudes identitaires essentiellement fondées, dans son discours, sur la foi catholique et des valeurs traditionnelles ${ }^{30}$ ». Et, pour maintenir cet ordre, le radicalisme d'un Papineau, sans être caché sous le boisseau, doit être, parmi tant d'autres éléments, neutralisé. Le père

26 Camille Roy, Manuel d'histoire de la littérature canadienne de langue française, Montréal, Librairie Beauchemin limitée, 1939, p. 31-32.

27 Camille Roy, Histoire de la littérature canadienne, Québec, Imprimerie de l'Action sociale limitée, 1930, p. 41.

28 Lucie Robert, Discours critique et discours historique dans le Manuel d'histoire de la littérature canadienne de langue française de $M^{g r}$ Camille Roy, p. 71.

29 Il écrit ailleurs, dans l'édition de 1918: «[L]a rhétorique est dangereuse pour les poètes: elle les expose à d'incessantes banalités, surtout quand elle chante la patrie et ses gloires traditionnelles. Seule une originalité forte peut triompher de ces tentatives d'enfler la voix, d'étonner le lecteur par des mots grandiloquents, par de sonores développements.» Cité par Karine Cellard, Leçons de littérature, p. 70. Ibid., p. 205. 
Baillargeon y veillera en intégrant la parole de Papineau à son manuel et en contrôlant ainsi l'analyse. Une "nécessité pour éviter le pire», disait Lucie Robert.

Papineau se voit ainsi consacrer une section complète du manuel, sous-titrée «L'idole d'une génération exaltée». Une biographie permet de suivre son parcours politique. On s'attache également à l'éloquence de l'homme, à ce «physique exceptionnel d'orateur ${ }^{31}$ ", à ses grands dons intellectuels. Baillargeon va même jusqu'à reproduire un long extrait du discours de Saint-Laurent ${ }^{32}$, qui remonte à mai 1837. Le texte est, dans ce cas-ci, tiré de l'Histoire des patriotes ${ }^{33}$ de Gérard Filteau, parue en 1938-1939, qui offre elle-même une lecture plutôt nationaliste et conservatrice des événements ${ }^{34}$.

Le choix de l'extrait par le père Baillargeon permet d'en baliser l'analyse, de relever au passage ce qu'il croit être des débordements, des effets de cape, des faussetés, même. Il écrit ainsi: "Le discours de Papineau devient amer, fielleux.» Ou encore: "Allusion sinistre au régicide de Cromwell: l'orateur dépasse la mesure.» Le père Baillargeon parle également de «l'allure démagogique de la phrase (expressions outrées, termes grandiloquents)», de la "preuve tendancieuse et spécieuse ${ }^{35} »$. De nouveau, le paradoxe est révélé, comme chez $\mathrm{M}^{\mathrm{gr}}$ Camille Roy: la parole enflammée et débordante est paradoxalement contrainte à une sorte d'affaiblissement qui s'accentue avec le temps. Baillargeon coupe le lien avec ce passé qui s'est éteint: "Que Papineau ait été un grand orateur, ses contemporains le redisent à l'envi et ses discours, même privés du prestige de ses dons physiques, le rappellent un peu ${ }^{36}$.» Façon de dire à peine plus pour faire entendre beaucoup moins...

Balisé par la lecture de Baillargeon, le texte de Papineau, désincarné, s'appauvrit irrémédiablement; la valeur des mots est prisonnière du passé - seuls les contemporains pouvaient juger de la force du tribun. On y reconnaîtra, de nouveau, cette stratégie du dessèchement, de la parole devenue muette parce que privée de corps. Berthelot Brunet l'avait bien dit dans son Histoire de la littérature canadiennefrançaise, qui semble condenser les idées reçues sur l'homme politique: les «morceaux que l'on rapporte» de Papineau sont «une lave bien refroidie ${ }^{37}$ ».

Chez Baillargeon, on opposera, encore, les figures de Papineau et de La Fontaine: "Là où Papineau a échoué, La Fontaine a brillamment réussi ${ }^{38}$.» Comme pour enfoncer le clou, l'auteur donne à lire un discours de La Fontaine, tiré du Cours d'histoire du Canada ${ }^{39}$ de Thomas Chapais. Son titre est sans ambiguïté: "Discours contre Papineau». Lucie Robert rappelait cette stratégie consistant à «sélectionner

31 Samuel Baillargeon, Littérature canadienne-française, préface de M. le chanoine Lionel Groulx, Montréal, Fides, 1957, p. 42.

32 Le père Baillargeon parle étrangement de «Saint-Laurent sur Richelieu»...

33 Gérard Filteau, Histoire des Patriotes, Montréal, Éditions de l'A.C.-F., 1938-1939, trois tomes.

34 Voir Marie-Frédérique Desbiens, "Le centenaire des rébellions. "Des patriotes au goût du jour"», Yvan Lamonde et Denis Saint-Jacques (dir.), 1937 : un tournant culturel, Québec, Presses de l'Université Laval, coll. «Cultures québécoises», 2009, p. 134-135.

35 Samuel Baillargeon, Littérature canadienne-française, p. 45.

36 Ibid.

37 Berthelot Brunet, Histoire de la littérature canadienne-française, p. 20.

38 Samuel Baillargeon, Littérature canadienne-française, p. 47.

39 Thomas Chapais, Cours d'histoire du Canada, vol. VI: 1847-1851, Québec, Librairie Garneau, 1933, 363 p. 
des écrits qui souvent [...] nuisent» aux radicaux. Il s'agit, ici, de la longue réponse que La Fontaine avait servie à Papineau lors de leur confrontation au parlement du Canada-Uni, à Montréal, le 23 janvier 1849. Comme pour le texte de Papineau, Baillargeon en balise la lecture, mais donne plutôt à découvrir ici un homme mesuré qui vient à bout des plus grands discours. Ainsi: "Il répondit en un discours posé, où la force logique pulvérise méthodiquement l'adversaire ${ }^{40}$.» Là où l'analyse de Baillargeon parlait de débordement, de sarcasmes et d'arguments spécieux chez Papineau, elle tient, sur La Fontaine, un discours symétrique, parfaitement - et délibérément, sans doute - contraire:

Le logicien implacable a composé un tissu serré d'arguments. Par de courtes preuves ironiques, multipliées habilement, l'orateur accule froidement son adversaire au pied du mur. Pas d'invectives, pas de termes grandiloquents, mais l'affirmation solide et puissante d'un homme sûr de sa position, maître de ses actes et au courant du jeu de son adversaire ${ }^{41}$.

La présence de Papineau, ailleurs dans le manuel, confirme cette opposition et le place, de nouveau, du côté du faux, du mensonge, même. L'homme politique est ainsi le sujet d'un autre extrait de texte, qui illustre la pensée et le parcours de Benjamin Sulte (1841-1923). Ce dernier ne trouve que peu de grâce aux yeux de Baillargeon: peu fiable et sans méthode historique, son anticléricalisme le rapproche du "socialisme» (!) ; il y a, chez lui, « déviation» de l'esprit. Le «portrait de Papineau » que Sulte réalise dans ses Mélanges historiques et que reproduit le père Baillargeon permet, par une sorte de contamination croisée, de confirmer les jugements respectifs sur ces deux hommes. Dans cette perspective, ce que Sulte dira de positif et de bien sur Papineau deviendra, escorté par les propos de Baillargeon, suspect; ce que Sulte dira et qui se rapprochera des propos de Baillargeon renforcera ces derniers, au sens où même un mécréant osera dire ce qu'un rédemptoriste affirmera des années plus tard. Le discrédit est sélectif, en somme. Et, même chez un «socialiste» comme Sulte, on retrouve la bonne vieille distinction entre la voix et la plume:

Nous avons peu d'hommes qui se donnent la peine de travailler pour maîtriser la langue écrite; nous en avons encore moins qui apprennent à parler selon l'art, soit devant un auditoire, soit dans un salon. Papineau excellait dans ces deux derniers genres; mais quand il prenait la plume, on ne le retrouvait pas à la même hauteur; pourtant il écrivait fort bien en tant qu'il s'agit de faire comprendre les idées que l'on émet. Le style de l'écrivain lui manquait. Il était maître du style de l'orateur qui utilise la voix, le geste, la circonstance de lieu, à part le fond de la pensée, tandis que l'écrivain n'a à sa disposition que des mots tracés en noir sur un fond blanc pour exprimer tout ce qu'il veut faire entendre; $c^{\prime}$ est plus difficile ${ }^{42}$.

40 Samuel Baillargeon, Littérature canadienne-française, p. 45.

41 Ibid., p. 48.

42 Benjamin Sulte, Mélanges historiques, cité par Samuel Baillargeon, Littérature canadienne-française, p. 77. 
La parole de Papineau, à l'heure du néonationalisme et tandis que l'étreinte du clérico-nationalisme se desserre rapidement, est-elle reçue différemment? Comme l'a constaté l'historien Serge Gagnon dès 1966, suivi par Jean-Paul Bernard en $1983^{43}$, il y a bien un retour des patriotes dans le discours social et dans la mémoire littéraire des années 1960, comme en témoignent notamment les rééditions de l'Histoire de l'insurrection au Canada ${ }^{44}$ et la parution de La victoire de Saint-Denis ${ }^{45}$ (1968) par Robert-Lionel Séguin, seul titre de la collection du «Centrentenaire» des Éditions Parti pris, qui veulent célébrer autre chose que le centenaire de la Confédération canadienne. La décennie suivante verra le même engouement pour ces patriotes. La désignation par le gouvernement Lévesque du boulevard Richelieu, entre Iberville et Saint-Ours, comme "chemin des Patriotes», le 24 juin 1977, n'en est qu'un exemple parmi tant d'autres ${ }^{46}$. Certains, comme l'historien Jean-Paul de Lagrave, verront dans tous ces phénomènes une récupération politique: «Dans le cas des patriotes de 18371838, un travail de récupération est en cours qui les donne comme ancêtres à ceux qui prônent l'indépendance du Québec, que ce soit dans la clandestinité ou dans la légalité ${ }^{47}$.»

Ce retour en force pave-t-il la voie à une nouvelle appréciation du chef politique de l'époque des patriotes, du moins idéologiquement? Ce n'est pas tant que l'histoire littéraire accepterait enfin la littérarité de ses textes, si tant est que cette dernière existe réellement. Mais on peut croire que les critiques idéologiques, instillées dans les propos littéraires de $\mathrm{M}^{\mathrm{gr}}$ Camille Roy et de Samuel Baillargeon, pourraient disparaître, d'autant que les raisons idéologiques qu'avait le clergé de neutraliser la charge radicale de Papineau semblaient beaucoup moins prégnantes au milieu des années 1960. Papineau apparaissait même, pour certains, comme un prédécesseur du Québec moderne, du nationalisme puis de l'indépendantisme. Gérald Godin n'écrivait-il pas, à la faveur de la victoire péquiste de 1976: "prendre en main leur gouvernement, on appelle ça, dans le gouvernement actuel du Québec: réaliser la souveraineté. D'où l'affirmation qu'il y a filiation entre Louis-Joseph Papineau et le Parti québécois ${ }^{48}$ » ? Une idéologie en a-t-elle chassé une autre?

Retournons, de nouveau, aux textes et attachons-nous au Manuel de littérature canadienne-française de Roger Duhamel et à l'Histoire de la littérature française du Québec dirigée par Pierre de Grandpré. Duhamel, qui sera directeur du

43 Serge Gagnon, «Pour une conscience historique de la révolution québécoise», Cité libre, n 83, janvier 1966, p. 9; Jean-Paul Bernard, «La mémoire actuelle des patriotes», Jean-Paul Bernard (dir.), Les rébellions de 1837-1838. Les patriotes du Bas-Canada dans la mémoire collective et chez les historiens, Montréal, Boréal Express, 1983, p. 11-12.

44 Montréal, Orphée, 1963 et 1968; Montréal, Leméac, 1968 (comprenant une introduction d'Hubert Aquin); Montréal, Réédition-Québec, 1968.

45 Robert-Lionel Séguin, La victoire de Saint-Denis, Montréal, Éditions Parti pris, 1968, 45 p.

46 Michel Girard, «Entre Iberville et Saint-Ours : le boulevard Richelieu sera désigné le chemin des Patriotes», La Presse, 23 juin 1977, p. 13.

47 Jean-Paul de Lagrave, "La récupération des patriotes», Le Devoir, 3 décembre 1979, p. 4.

48 Gérald Godin, «La démocratie venue du Sud» [1979], Écrits et parlés, t. II : Politique, édition préparée par André Gervais, Montréal, l’Hexagone, coll. «Itinéraires», 1993, p. 204. 
Montréal-Matin, professeur de littérature à l'Université de Montréal et diplomate, construit une histoire plutôt traditionnell ${ }^{49}$ : « [C]'est en esthète et non en sociologue qu'il aborde le corpus québécois, dans une brève histoire littéraire où le sens des hiérarchies informe à la fois le discours critique et les modalités de classement ${ }^{50}$.» Pierre de Grandpré, de son côté, a été le condisciple de Pierre Vadeboncœur et de Pierre Elliott Trudeau au collège Jean-de-Brébeuf; critique littéraire, romancier, haut fonctionnaire, l'homme dirige le projet des éditions Beauchemin, fort ambitieux (plus d'une vingtaine de collaborateurs). Cellard, après avoir rappelé les aléas de la préparation des quatre volumes, conclut d'ailleurs: «Courtepointe d'études menées au gré de plumes et de méthodes pour le moins variées, l'Histoire de la littérature française du Québec parvient difficilement à trouver une cohérence d'ensemble qui transcende la diversité de ses composantes ${ }^{51}$.» Si De Grandpré et Duhamel ne sont pas du même acabit et si les contextes sont fort différents, ils offrent pourtant un portrait de Papineau assez semblable, comme si, encore une fois, l'aiguille de la boussole n'avait pas beaucoup bougé depuis le manuel de $\mathrm{M}^{\mathrm{gr}}$ Camille Roy.

Reprenant presque mot à mot les propos de Samuel Baillargeon, Duhamel insiste à son tour sur la "lave refroidie» que sont les discours politiques de Papineau: «Il est souvent malaisé de relire les textes d'un tribun, parce qu'il aborde des actions d'une actualité périmée, parce qu'il s'abandonne au feu de l'inspiration, de l'improvisation. Papineau, malgré ses dons naturels, n'échappe pas à cette règle ${ }^{52}$.» La "règle» a bien quelque chose de spécieux: l'essai qui s'attacherait à un thème d'actualité ne serait-il pas, ainsi, toujours caduc? Aussi, les «dons naturels» de l'homme politique, lieu commun de la fortune mémorielle de Papineau, ne le sauvent pas, bien au contraire. L'homme n'a pas eu à faire d'efforts, et ce, dès son jeune âge, comme le disait déjà le père Baillargeon: «Écolier brillant, mais piètre travailleur, il accède sans effort au premier rang de sa classe ${ }^{53}$.» Cette facilité de l'homme génial, répétée sans cesse dans ces histoires littéraires, place l'homme politique à l'écart (stratégie d'isolement du reste de la société), du côté d'une nature généreuse qui en fait un être d'exception. Quel mérite peut-il y avoir de ce côté? Son propos ne peut avoir de répercussions sur une longue durée, puisqu'improvisé, puisqu'émanant d'une puissance naturelle qui s'éteint, inexorablement, avec le décès de l'homme.

De leur côté, dans le chapitre XV («L'éloquence [1830-1860]») du premier volume de l'Histoire de la littérature française du Québec, Pierre de Grandpré et Arsène Lauzière, professeur au Collège militaire de Kingston, s'attachent à la figure de Papineau. Le topos de la «lave refroidie» structure même le fil narratif: la "chaleur» de l'improvisation de Papineau, une "pensée inégale, qui se fait au chaud", sa façon de "méduser et de galvaniser les foules» dans le "feu de l'action», cela finit par tout embraser (Papineau est «comme brûlé par son propre souffle incendiaire»)

\footnotetext{
49 Karine Cellard, Leçons de littérature, p. 235.

50 Ibid.

51 Ibid., p. 246.

52 Roger Duhamel, Manuel de littérature canadienne-française, Montréal, Éditions du Renouveau pédagogique, 1967, p. 10.

53 Samuel Baillargeon, Littérature canadienne-française, p. 41.
} 
avant que le propos ne devienne complètement froid («[é]lectrisante à la tribune, sa pensée est glacée, aujourd'hui ${ }^{54}$ »). La portée radicale de ses mots, quand ils passent à l'écrit, est tout aussi gelée, inanimée. Les écrits de Papineau - les deux auteurs mentionnent, au passage, l'Histoire de l'insurrection du Canada - donnent à voir un «écrivain sans vocation particulière ${ }^{55}$ ».

Autre façon d'isoler Papineau, bien connue depuis le manuel de Camille Roy: la comparaison désavantageuse avec Étienne Parent et Louis-Hippolyte La Fontaine. Dans une note en bas de page, Grandpré et Lauzière insistent sur la pensée mesurée du plus grand des orateurs, qui n'est pas Papineau:

\begin{abstract}
À lire ou à relire seulement ce que nous ont légué ces anciens orateurs, le plus grand parmi eux - sûrement le plus modeste - par son métier de joindre l'esprit de réflexion à l'émotion contrôlée, ce n'est pas Papineau, mais Étienne Parent, dont certains articles de journaux eussent ajouté à sa gloire s'il les eût prononcés à la tribune avant de les faire imprimer ${ }^{56}$.
\end{abstract}

Étrange renversement: la parole à la tribune devient, tout à coup, gage de postérité, tandis qu'elle engendrait chez Papineau une surchauffe avant l'extinction du propos.

En outre, comme le père Baillargeon, Lauzière et Grandpré reproduisent l'intervention parlementaire du 23 janvier 1849 de La Fontaine, comme s'il s'agissait du principal morceau de bravoure de l'homme politique. De nouveau, ce passage ne semble pas tant choisi pour montrer l'efficacité de la «dialectique de juriste» que pour reprendre les attaques contre l'inconséquence de Papineau, pour les réactiver, même. Rien n'a vraiment changé.

\title{
PAPINEAU L'ÉCRIVAIN, AUJOURD'HUI
}

L'idéologie clérico-nationaliste a-t-elle fini par s'imposer et essentialiser des critiques et des idées reçues sur le "cas» de Louis-Joseph Papineau? Tout se passe en effet comme si l'image de Papineau était à peu près demeurée inchangée, de 1918 à 1967. Et même au-delà: les réflexions d'ordre littéraire sur Papineau se doublent encore aujourd'hui, bien souvent, des mêmes critiques idéologiques. Les entreprises récentes d'histoires littéraires, fruits du travail de spécialistes universitaires, éloignées autant que faire se peut des idéologies d'antan - à moins que d'autres se soient subrepticement imposées ${ }^{57}$-, en témoignent éloquemment. Â commencer par le volume de la

54 Pierre de Grandpré (dir.), Histoire de la littérature française du Québec, t. I: 1534-1900, Montréal, Librairie Beauchemin limitée, 1967, p. 150.

55 Ibid.

56 Ibid., p. 149.

57 Voir, notamment, à propos des fondements nationalistes de l'entreprise du Dictionnaire des œuvres littéraires au Québec, Jonathan Livernois, «Papineau et le libéralisme du 19e siècle à l'aune de l'indépendantisme québécois des années 1970 : le rôle du Dictionnaire des œeuvres littéraires du Québec», Chantal Savoie (dir.), avec la collaboration de Mylène Bédard, Le Dictionnaire des œuvres littéraires du Québec, témoin et acteur de l'essor des études littéraires québécoises, Québec, Nota bene, coll. «Séminaires», 2015, p. 81-110. 
collection «Que sais-je?» (1974) consacré à la littérature québécoise, que son auteur, Laurent Mailhot, a largement augmenté en 1997 et fait paraître chez Typo. On sait que le professeur de littérature de l'Université de Montréal a eu le mérite, au milieu des années 1970, de mettre en relief l'intérêt des lettres et écrits des patriotes. Cela ne l'empêche pas de juger dans son ouvrage que les textes de Papineau «déçoivent». Il ajoute ce commentaire, curieux: son «lieutenant, qui deviendra son adversaire, La Fontaine, est plus posé: "le Poincaré de ce Briand" ${ }^{8}$ ». Quel est l'intérêt d'opposer de nouveau, dans ce contexte, Papineau à La Fontaine, en prenant soin - par la comparaison empruntée à Berthelot Brunet ${ }^{59}$ - de déprécier le premier? Le politique s'immisce ici dans les interstices du discours littéraire, sans qu'il y ait, en apparence, de raisons qui expliqueraient un tel jugement.

Dans le deuxième volume (1992) de La vie littéraire au Québec, entreprise colossale née en 1989 de la cuisse du Dictionnaire des cuvres littéraires du Québec, où Papineau n'avait guère la part belle ${ }^{60}$, on reconnaît certes, dès les premières pages de l'ouvrage, un statut d'écrivain à Papineau ${ }^{61}$. Le collectif, dirigé alors par Maurice Lemire, identifie même, dans certains de ses discours, un «souci évident de littérarité $^{62}$ ». Mais on précise aussi:

\begin{abstract}
Papineau, malgré toute son information, ne s'affirme pas comme penseur original dans ses écrits. Il devait exceller dans l'improvisation, dans les réparties à brûlepourpoint, dans les joutes oratoires, mais pas devant la feuille blanche. [...] Au cours de sa longue retraite, Papineau aurait pu, à l'instar de Cicéron, retravailler ses discours pour en faire de véritables œuvres littéraires. Mais l'homme politique, semble-t-il, ne tient pas à entrer dans l'histoire en tant qu'écrivain. Aussi ne réviset-il pas non plus sa correspondance ${ }^{63}$.
\end{abstract}

Sort-on du registre littéraire lorsqu'on avance, également, que son argumentation n'est pas «apodictique» et qu'il est un homme politique qui «se laisse porter par la vague plus qu'il ne la provoque ${ }^{64}{ }^{\prime}$ ? Malgré un portrait que l'on veut pondéré, on parle de son manque d'originalité, de sa méprise face à l'enthousiasme des Étatsuniens à soutenir la rébellion prochaine. On rappelle ses «arguments souvent réducteurs» qui «amènent les auditeurs aux conclusions qu'il désire ${ }^{65}$ ». Plus encore, comme dans le bon vieux temps, l'opposition avec Parent est réactivée, dépassant largement le seul cadre littéraire:

58 Laurent Mailhot, La littérature québécoise depuis ses origines, Montréal, TYPO, 1997, p. 32.

59 Berthelot Brunet, Histoire de la littérature canadienne-française, p. 21.

60 Voir Maurice Lemire (dir.), Dictionnaire des œuvres littéraires du Québec, Montréal, Fides, 1978-2011, huit tomes. Voir également Jonathan Livernois, «Papineau et le libéralisme du 19e siècle à l'aune de l'indépendantisme québécois des années 1970: le rôle du Dictionnaire des œuvres littéraires du Québec», p. 100-105.

61 Maurice Lemire (dir.), La vie littéraire au Québec, t. II : 1806-1839: le projet national des Canadiens, Québec, Presses de l’Université Laval, 1992, p. 3.

62 Ibid., p. 242.

63 Ibid., p. 84.

64 Ibid.

65 Ibid., p. 243. 
Papineau doit son incontestable renommée plus à ses dons oratoires qu'à ses qualités d'homme politique. Dans une civilisation encore largement dominée par l'oral, il a trouvé le moyen d'atteindre le plus grand nombre de personnes. Le journal, au contraire, s'adresse encore à une population restreinte. C'est pourquoi Parent, avec des idées plus justes et des arguments mieux construits, exerce cependant moins d'influence ${ }^{66}$.

Toutes ces critiques ne sont pas sans rappeler celles, extrêmement sévères, de l'historien Fernand Ouellet qui, au cours des années 1950 et 1960, ont marqué d'une pierre blanche l'historiographie sur Papineau. Les propos du second volume de La vie littéraire au Québec ne semblent guère ébranler le régime d'interprétation de l'œuvre et du parcours de Papineau.

Dernière borne du parcours, de laquelle le lecteur actuel n'a pas une grande distance: l'Histoire de la littérature québécoise, de Michel Biron, François Dumont et Élisabeth Nardout-Lafarge, parue en 2007. La présentation de Papineau y est des plus pondérées, accompagnée de deux extraits - l'un de son «discours-fleuve prononcé à l'occasion de la mort du roi d'Angleterre George III $^{67}$ ", et l'autre de son «Discours de Saint-Laurent» - qui permettent de bien voir à l'œuvre l'évolution de sa pensée. On rappelle "sa verve exceptionnelle, souvent empathique ${ }^{68}$ ", que les extraits choisis ne permettent guère de deviner, par contre. Ce qui frappera, sans doute, c'est, encore une fois, l'absence de référence aux textes de Papineau (comme l'Histoire de l'insurrection), sinon pour dire qu'il a collaboré "à divers journaux socialistes ${ }^{69}$ » en France. L'homme n'est encore qu'un orateur. Et il y a bien quelque chose du déclassement dans une telle phrase: "Plus encore par l'éloquence de tribuns comme Papineau, $c^{\prime}$ est par la création de journaux que s'élabore et se diffuse une nouvelle conscience politique $^{70}$.» L'écrit l'emporte encore.

Comment expliquer cette situation, cette persistance de vieilles critiques idéologiques au cœur de l'histoire littéraire qui, croyait-on, avait nettoyé les écuries d'Augias d'une critique clérico-nationaliste, lestée d'objectifs autres que littéraires? On peut proposer, peut-être, deux explications, complémentaires. L'une est plus lourde que l'autre et touche l'identité québécoise.

On peut d'abord pointer du doigt ce que Micheline Cambron, dans des travaux récents, définissait comme la «non-lecture» des textes du xIX siècle. Il existe, selon elle, une sorte de vulgate, immuable, qui reconduit toujours les mêmes idées reçues sur la période, comme cette impression que «les œuvres littéraires - et à l'époque, cela inclut les textes journalistiques, les textes historiographiques et les discours présents dans l'espace public — seraient sans intérêt du point de vue de la forme et ne

66 Ibid., p. 245.

67 Michel Biron, François Dumont et Élisabeth Nardout-Lafarge, avec la collaboration de Martine-Emmanuelle Lapointe, Histoire de la littérature québécoise, Montréal, Boréal, 2007, p. 65.

68 Ibid., p. 64.

69 Ibid., p. 65

70 Ibid., p. 66. 
vaudraient que pour leur intérêt documentaire ${ }^{71} »$. Les études littéraires n'y auraient rien changé? Cambron parle en tout cas d'un «échec du discours savant, la sédimentation des discours n'ayant pas mené à une transformation des savoirs mais plutôt à une réitération, involontaire bien sûr, de la vulgate ${ }^{72}$ ». S'attachant aux lectures de Jean Rivard ${ }^{73} \mathrm{~d}^{\prime}$ Antoine Gérin-Lajoie, elle montre que «le jugement sur cette œuvre, largement diffusé sur divers supports (revue, journal, livre, manuel, bande dessinée), a fait consensus à partir des premières suggestions de l'abbé Casgrain, en 1872, jusque dans les années 1980, période où cette interprétation a été contestée ${ }^{74}$ ». À l'en croire, les jugements du prédécesseur sont souvent repris tels quels par le successeur, sans relecture de l'œuvre. Il y a là, dit Cambron, «le poids des reprises et des citations dans la réduction de la diversité des lectures et de la mise en jeu du sens ${ }^{75}$ ». La question demeure pendante: a-t-on vraiment lu Papineau? La frontière de la littérarité n'est pas fixée définitivement, et le parti des relectures des œuvres du XIX siècle, comme celles que propose Micheline Cambron, devrait être pris, même dans le cas de Papineau.

Si la persistance de vieux traits dans les propos tenus sur Papineau en tant qu'écrivain peut s'expliquer par un vice de la profession ou de la discipline, force est de constater qu'il y a une autre explication, dont les ramifications sont plus étendues et, surtout, plus profondes. Si les stratégies sont demeurées, c'est peut-être que le problème de fond n'a guère changé: négocier l'héritage de Papineau, par trop révélateur de la situation canadienne-française puis québécoise. Yvan Lamonde le proposait, en tout cas, dans son étude récente sur l'homme politique: «En étant le représentant d'un inachèvement et le bouc émissaire d'un inaccomplissement, Papineau est devenu le porteur des déceptions, des défaites, des frustrations et l'image exacte, si l'on peut dire, de l'ambivalence d'un destin et d'une représentation de $\mathrm{soi}^{76}$.» Une sorte de miroir, en somme, sur lequel les Québécois ne peuvent s'empêcher de jeter un regard oblique. Papineau ne saurait être oublié, mais sa charge mémorielle est peut-être trop lourde, trop difficile à négocier. Cela permettrait d'expliquer que ce poids symbolique soit mis entre parenthèses. La littérature et les récits que l'on en fait, au Québec, créeraient de ces parenthèses, restées les mêmes grâce à des stratégies pérennes d'inclusion et d'exclusion de l'homme politique réduit à un homme de discours, éteints depuis longtemps.

Du clérico-nationalisme à ce qu'est le temps présent, l'idéologie a peut-être changé, mais le problème du nœud interprétatif de ce que Papineau représente et a représenté demeure bien fiché, entre les lignes du texte. La tâche dépasse les études littéraires, probablement.

71 Micheline Cambron, «La non-lecture du 19e siècle québécois ou la constitution d'une vulgate», projet de recherche, en ligne sur le site du CRILCQ: http://www.crilcq.org/projet/la-non-lecture-du-xixe-sieclequebecois-ou-la-constitution-dune-vulgate (page consultée le 27 septembre 2016).

72 Ibid.

73 Antoine Gérin-Lajoie, Jean Rivard, le défricheur. Récit de la vie réelle, Montréal, J.-B. Rolland et fils, libraires-éditeurs, 1874 et 1876.

74 Micheline Cambron, "Lecture et non-lecture de Jean Rivard d'Antoine Gérin-Lajoie», Karine Cellard et Martine-Emmanuelle Lapointe (dir.), Transmission et héritages de la littérature québécoise, Montréal, Presses de l’Université de Montréal, coll. «Espace littéraire», 2011, p. 114.

75 Ibid., p. 137.

76 Yvan Lamonde, Fais ce que dois, advienne que pourra. Papineau et l'idée de nationalité, Montréal, Lux éditeur, coll. «Mémoire des Amériques», 2015, p. 9-10. 